\title{
Influencia de la edad y el tiempo de madurez en la capacidad de retención de agua (CRA) en la carne de ovino criollo
}

\author{
Influence of age and time of maturity in the water retention capacity (CRA) in the Lamb meat \\ criollo
}

\author{
Trujillo N. Yanine Y.*, Ayala M. Magda M., Duran O. Daniel S.
}

Universidad de pamplona. Facultad de ingenierías y arquitectura, Departamento de alimentos, Grupo de investigación GINTAL. Universidad de Pamplona, ciudadela universitaria, barrio el buque, Pamplona, Norte de Santander Colombia

Recibido 09 Febrero 2015; aceptado 22 de Marzo 2015

\section{RESUMEN}

La capacidad de retención de agua (CRA) es una propiedad físico-química importante en la calidad de la carne ovina, esta se encuentra relacionada con el color, firmeza, jugosidad y textura de la carne cocida. Una baja capacidad de retención de agua puede ocasionar perdidas de algunas proteínas solubles, vitaminas y minerales, además de afectar la jugosidad en la carne cocida. Por tal razón, el objetivo de este trabajo fue establecer la capacidad de retención de agua en la carne de ovino criollo, sacrificado a diferentes edades, madurado bajo diferentes tiempos. Para ello, se sacrificaron 36 ovinos enteros obtenidos de múltiples cruces entre razas Corriedale y Romney Marsh, los cuales fueron previamente sometidos a un ayuno de 12 horas. Las 36 canales obtenidas fueron empacadas en bolsas de polipropileno de baja densidad y llevadas a refrigeración a $4 \pm 2^{\circ} \mathrm{C}$ para su maduración a tres tiempos (24h, $48 \mathrm{~h}$ y $\left.72 \mathrm{~h}\right)$. Finalizado el tiempo de maduración respectivo, se obtuvo de cada canal el corte rack (5-9 costilla), del cual se evaluó la capacidad de retención de agua. Como resultado se obtuvo que la edad al sacrificio y el tiempo de madurez afectarón la capacidad de retención de agua, estableciendo una relación directa entre el aumento de la edad y la $C R A$, asimismo una mayor afectación del tiempo de madurez (48h y 72h) en animales jóvenes sobre este parámetro.

Palabras clave: Agua, edad, madurez, ovino, retención

\section{ABSTRACT}

The water holding capacity (CRA) is an important quality of sheep meat physicochemical property, this is related to the color, firmness, juiciness and texture of cooked meat. A low water holding capacity can cause losses of certain soluble proteins, vitamins and minerals, in addition to affecting the juiciness. For

*Autor a quien debe dirigirse la correspondencia. E-mail: yaninetrujillo@unipamplona.edu.co this reason, the aim of this study was to establish the capacity of water retention in 
the Creole sheep meat sacrificed at different ages, grown under different times. To do this, 36 whole sheep obtained from multiple crosses between races Corriedale and Romney Marsh, which were previously subjected to fasting for 12 hours were sacrificed. The 36 channels obtained were packed in bags of low density polypropylene and carried refrigeration at $4 \pm 2^{\circ} \mathrm{C}$ to mature three times $(24 \mathrm{~h}, 48 \mathrm{~h}$ and $72 h$ ). After the respective time of ripening, it was obtained from each channel cut rack (5-9 rib), which the water holding capacity was evaluated. As a result it was found that the age at slaughter and the time of maturity affected the water holding capacity, establishing a direct relationship between increasing age and CRA also greater involvement time of maturity (48h and $72 h$ ) in young animals this parameter.

Keywords: Water, age, maturity, sheep, retention

\section{INTRODUCCIÓN}

La capacidad de retención de agua fue definida por ZHANG et al., (2005), como la habilidad de la carne para retener su contenido acuoso durante la aplicación de fuerzas externas como pueden ser, corte, calentamiento, picado o presión. En (1983), Offer y Tricrick, mostraron evidencias de que la mayor parte del agua que se encuentra directamente relacionada con la CRA, se encuentra en el musculo retenida por fuerzas capilares entre los filamentos finos y los filamentos gruesos de miofibrillas. Onega et al., (2001), determinaron que la carne cruda de cordero se caracteriza por presentar un color rojo brillante e intenso, un nivel moderado de grasa infiltrada y un débil aroma a suero, siendo esta una carne tierna y con escasa exudación gracias a su elevada CRA. Según Lawrie y Ledward (2006), la evaluación de la calidad de la carne se debe realizar a partir de los parámetros de apariencia, jugosidad, terneza y sabor, siendo estos afectados directamente por la CRA, además autores como Zhang et al., (2005), la relacionan con la pérdida de peso principalmente por liberación de jugos. Díaz Diaz-Chiro (2001), determinaron que la CRA de la carne está relacionada con la textura, terneza, color de la carne cruda y la jugosidad de la carne cocida, la cual se encuentra en dos estadios una inicial la cual produce sensación de humedad al inicio de la masticación siendo esta dependiente de la CRA. Esta retención de agua se produce a nivel de las cadenas de actino-miosina; la segunda que corresponde a una jugosidad continúa depende de la cantidad de grasa que posea la carne. Por otra parte la CRA de la carne está relacionada con el color de la carne cruda de los mamíferos inmediatamente después del sacrificio los cuales contienen, por término medio, un $75 \%$ de agua (Lawrie, 1998), porcentaje que varía con la especie de procedencia y el músculo que se considere, parte de este agua se pierde por evaporación durante el enfriamiento de las canales en corderos lechales estas pérdidas pueden llegar a ser de un $5 \%$. En este orden de ideas la literatura reporta que el comportamiento de la CRA puede estar afectado por el desarrollo fisiológico de los músculos y por el aumento de la grasa con la edad, a medida que este aumenta o disminuye la combinación de humedad y proteína se desplaza en dirección opuesta, convirtiendo a los cambios de la CRA en indicadores muy sensibles de los cambios en las proteínas miofibrilares (Hönikel, 1998). A su vez la edad se encuentra relacionada con la perdida de terneza de la carne, debido al incremento del número de uniones de las moléculas de tropocolágeno. Por otra parte la carne es considerada el resultado de una serie de 
transformaciones estructurales y de reacciones bioquímicas que tienen lugar en el musculo tras la muerte del animal. Durante el proceso de maduración post-mortem, se producen cambios que afectan su calidad fisicoquímica, tecnológica y sensorial. Este momento corresponde al proceso de conversión del musculo en carne el cual está compuesto por tres fases según (Sentadreu et al., 2002): fase pre-rigor durante la cual el musculó permanece excitable y se correspondería con la fase de supervivencia del sistema nervioso (Chrystall y Devine, 1985); el rigor, en la que los componentes energéticos (ATP, fosfocreatina, glucosa) se agotan; y por último la fase post-rigor de maduración en la que se produce una destrucción de la arquitectura muscular, ya que tras la muerte celular se produce una acidificación progresiva de la carne que determina un descenso en la carga eléctrica de las proteínas e induce a un incremento en su hidrofobicidad, presentando una reducción en su CRA (Ouali et al., 2013). Posterior al rigor mortis, comienza la etapa de tenderizacion que produce la mejora de la terneza de la carne como consecuencia fundamentalmente de la rotura de la estructura miofibrilar por parte de sistemas proteolíticos endógenos que juegan un papel determinante (Ouali 1992; Sentandreu et al., 2002; Weaver et al., 2009; Huff-Lonergan et al., 2010). Sin embargo, a pesar del conocimiento acumulado a lo largo de los años sobre los procesos que conducen a la instauración del rigor mortis y los que ocurren durante el periodo de maduración postrigor, no se ha conseguido explicar de forma definitiva la variabilidad en la tenderizacion de la carne, de forma que están surgiendo hipótesis que abren la puerta a nuevos campos de la investigación (Sierra, 2010). Estudios como el desarrollado por Martínez Cerezo et al., (2002), en el que trabajaron con las muestras de músculos semitendinosus, semimembranosus de ovinos razas Merino Español, Churra y aragonesa, aplicando 1, 2, 4, 8 y 16 días de maduración, cuyos resultados mostraron mejoras en la nota de terneza asignada por el test de consumidores conforme transcurrió la maduración desde 1 hasta 8 días. Kuber et al., (2003), realizaron estudios en el musculo longissimus dorsi de ovinos $7 / 8$ Columbia x 1/8 Dorset, aplicaron tratamientos de 1, 3, 6, 12, 24 y 48 días en animales portadores del gen callipyge y testigos. Registraron un efecto ablandador conforme transcurrió la maduración, particularmente en los corderos que no presentaban el gen que provoca la hipertrofia muscular. Relacionando las investigaciones mencionadas con los cambios en la textura de la carne de ovino se identifica que la capacidad de retención de agua, presenta una afectación con el cambio de $\mathrm{pH}$ que se presenta en la conversión del musculo en carne, estableciendo su mínima capacidad cuando se alcanza el punto isoeléctrico de las proteínas ( $\mathrm{pH} 5.0$ y 5.5), que es el pH último de la carne tras sufrir el rigor mortis. En este contexto el aumento de la CRA durante el tiempo de maduración puede tener explicación, en el incremento del $\mathrm{pH}$ muscular. No siendo esta la única variable que denota afectación sobre este parámetro, ya que autores como Melody et al., (2004), determinaron que la proteína titina puede formar enlaces con la miosina provocando un descenso en la solubilidad de las proteínas, en la terneza de la carne en el tamaño de las miofibrillas y en la CRA (Kim, 2010). Dado que la información que se encuentra sobre la carne ovina es muy reducida en nuestro país, este estudio tiene como objetivo determinar la influencia de la edad y el tiempo de madurez en la capacidad de retención de agua (CRA) en la carne de ovino criollo.

\section{MATERIALES Y MÉTODOS}

Este trabajo se realizó en el Edificio Antonio Betancourt Walker donde funciona el grupo de investigación en Ingeniería y Tecnología de Alimentos GINTAL de la Universidad de Pamplona.
Las muestras seleccionadas para el desarrollo experimental del proyecto fueron ovinos de raza criolla (machos enteros), procedentes de la ecoregión de García Rovira, que de acuerdo con el MARD en 2008 se encuentra posicionada a nivel de la región como zona de cría de ovinos. Se tomaron 36 animales después del destete (4 meses), que 
fueron confinados en la finca de un productor perteneciente a una asociación (CEPROGAR o ASOPACON), con el propósito de evitar la interferencia por las condiciones edafoclimaticas. A partir de los cuatro meses de edad, se sacrificaron 9 animales cada 2 meses (4, 6, 8 y 10 meses). Para el sacrificio, los ovinos fueron transportados a la Universidad de Pamplona en donde se sometieron a un ayuno de 12 horas (en aprisco). Transcurrido el tiempo de ayuno, los animales fueron sacrificados en la planta de derivados cárnicos de la Universidad, bajo condiciones higiénico sanitarias siguiendo los protocolos planteados por Albarracín en el 2013. Las canales, fueron empacadas en bolsas de polipropileno de baja densidad y la maduración se realizó en tres tiempos $(24,48$ y 72 horas), bajo condiciones de refrigeración $\left(4 \pm 2^{\circ} \mathrm{C}\right)$. Finalizando cada periodo de maduración se obtuvieron, los cortes comerciales de alto valor económico (rack o campana y silla refiriéndose al contenido de las 8 costillas superiores incluyendo el lomo).

El número de muestras para el análisis fue definido por:

$$
\begin{gathered}
N=4 E P \times 3 M D \times 3 R \\
N=36
\end{gathered}
$$

donde:

$\mathrm{N}=$ número de muestras empacadas a analizar

$\mathrm{EP}=$ número de edades y peso del animal

$\mathrm{MD}=$ número tiempos de maduración de la carne

$\mathrm{R}=$ número de repeticiones
La capacidad de retención de agua (CRA), fue determinada por triplicado aplicando el método propuesto por Plá et al., (2000), tomando exactamente $0,30 \mathrm{~g}$ de carne picada del corte rack, libre de grasa y tejido conjuntivo. Se colocó la muestra entre dos papeles de filtro Whatman $n^{\circ} 1$ de $55 \mathrm{~mm}$ de diámetro, previamente desecados, situados entre dos placas de vidrio de $9 \times 12 \mathrm{~cm}$ que se presionaron con un peso de $5 \mathrm{~kg}$ por $5 \mathrm{~min}$. Posteriormente, y tomando el papel filtro con la muestra de carne adherida, se midió la superficie de la mancha del agua expulsada y la de la carne con un pie de rey, y por diferencia entre el valor de ambas superficies, se obtuvo el valor del anillo del líquido ( $R Z)$.

La cantidad de agua extraída se calculó según la ecuación 1:

$\mathrm{mg}$ de agua $=\left(\mathrm{RZ}\right.$ en cm $\left.{ }^{2} / 0,0948\right) / 8$ Ecuación 1

La capacidad de retención de agua (CRA) se expresó como porcentaje de jugo expulsado.

Los resultados de la CRA fueron analizados estadísticamente empleando el método de análisis de la varianza ANOVA con un nivel de significancia de 0.05 , con el fin de determinar el efecto de la edad en la carne de ovejos sacrificados a los 4, 6, 8 y 10 meses y del tiempo de madurez (24h, $48 \mathrm{~h}$ y $72 h)$.

\section{RESULTADOS Y DISCUSIÓN}

El estudio evaluó la influencia de las variables independientes edad al sacrificio y tiempos de madurez sobre la CRA en la carne de ovinos criollos.

En la tabla 1 se muestra el comportamiento de la capacidad de retención de agua y el pH, medidos a las $24 \mathrm{~h}$ de sacrificio para las diferentes edades en estudio, la literatura reporta la edad como uno de los factores antemorten que afectan la calidad de la carne de ovino.
Tabla 1

Influencia de la edad sobre la capacidad de retención de agua y el $\mathrm{pH}$ en la carne de ovino

\begin{tabular}{|c|c|c|}
\hline Edad (meses) & $\begin{array}{c}\text { CRA } \\
\text { (\%jugo extraído) }\end{array}$ & $\mathbf{p H}$ \\
\hline 4 & $1,81 \pm 0,35^{\mathrm{a}}$ & $6,11 \pm 0,23^{\mathrm{a}}$ \\
\hline 6 & $1,65 \pm 0,15^{\mathrm{a}}$ & $5,79 \pm 0,28^{\mathrm{b}}$ \\
\hline 8 & $1,25 \pm 0,15^{\mathrm{b}}$ & $5,60 \pm 0,02^{\mathrm{b}}$ \\
\hline 10 & $1,17 \pm 0,05^{\mathrm{b}}$ & $5,75 \pm 0,25^{\mathrm{b}}$ \\
\hline $\boldsymbol{p}$-valor & 0,000 & 0,007 \\
\hline
\end{tabular}

$\mathrm{n}=36$. Media \pm Desv. Típica. Letras diferentes entre filas existe diferencia mínima significativa $p$-valor 0,05 
En este contexto se encontró que para la CRA entre la edad de 4 y 6 meses, los resultados son similares, al igual que entre la edad de 8 y 10 meses guardando la relación que a menor edad mayor porcentaje de jugo extraído por lo tanto, menor CRA, siendo consistentes con la literatura la cual describe, el aumento de este parámetro relacionado directamente con mayores edades. Asimismo se puede presumir un desarrollo fisiológico semejante para los dos bloques de edades.

En la tabla 1, se observa como la CRA presenta una relación inversa con el incremento del $\mathrm{pH}$ en relación con la edad, coincidiendo con el estudio realizado por Huff-Lonergan (2005), quien observo una relación estrecha entre la CRA y el $\mathrm{pH}$, indicando mayor habilidad de la carne para retener líquido a mayor edad de sacrificio, permitiendo la obtención de una carne valorada como más jugosa al ser cocinada. Resulta oportuno mencionar, que se estableció una relación entre el pH y la CRA, ya que como se presentó, cuando el pH se encuentra superior al 5,75 la cantidad de jugo extraído aumenta, por lo tanto la CRA estaría disminuyendo por encima de este valor. En este orden de ideas, los datos presentan el comportamiento planteado por Cañeque (2000), un descenso lento del pH con disminución de la temperatura de la canal, que no permite la desnaturalización de las proteínas y por ende la liberación de agua.

En la tabla 2, presenta Influencia del tiempo de maduración sobre la capacidad de retención de agua en la carne de ovino, parámetro postmortem que presenta influencia sobre las características físicas de la carne.
Tabla 2

Influencia del tiempo de maduración sobre la capacidad de retención de agua en la carne de ovino

\begin{tabular}{|c|c|c|c|c|}
\hline \multirow{2}{*}{$\begin{array}{c}\text { Edad } \\
\text { (meses) }\end{array}$} & \multicolumn{2}{|c|}{ CRA (\% jugo extraído) } & \multirow{2}{*}{ p-valor } \\
\cline { 2 - 4 } & $24 \mathrm{~h}$ & $48 \mathrm{~h}$ & $72 \mathrm{~h}$ & \\
\hline 4 & $1,85 \pm 0,27^{\mathrm{a}}$ & $2,01 \pm 0,56^{\mathrm{a}}$ & $1,87 \pm 0,17^{\mathrm{a}}$ & 0,541 \\
\hline 6 & $1,45 \pm 0,29^{\mathrm{a}}$ & $1,55 \pm 0,23^{\mathrm{a}}$ & $1,74 \pm 0,16^{\mathrm{b}}$ & 0,018 \\
\hline 8 & $1,12 \pm 0,07^{\mathrm{a}}$ & $1,05 \pm 0,15^{\mathrm{a}}$ & $1,19 \pm 0,06^{\mathrm{b}}$ & 0,010 \\
\hline 10 & $1,55 \pm 0,13^{\mathrm{a}}$ & $1,05 \pm 0,15^{\mathrm{b}}$ & $1,19 \pm 0,04^{\mathrm{c}}$ & 0,000 \\
\hline
\end{tabular}

$\mathrm{n}=36$. Media \pm Desv. Típica. Letras diferentes en filas existe diferencia $p$-valor 0,05

Como se observa en la tabla 2, la capacidad de retención de agua, presentó mayor liberación de jugo a las 72 horas de madurez para las edades de los animales más jóvenes 4 y 6 , encontrando que para la primera, los tiempos de madurez no presentan diferencia significativa, resultados que concuerdan con los estudios que reportan animales de corta edad con baja capacidad de retención de agua. La edad de 10 meses mostró la mayor capacidad de retención a las $24 \mathrm{~h}$ de madurez, la cual va disminuyendo en el tiempo, situación que puede relacionarse con la liberación por goteo de agua. Honikel (1988), determinó que este comportamiento se debe al cambio fisiológico presente en los sarcomeros y fibrillas contraídas después de la instauración del rigor y es debida a la reducción del espacio filamental. Por otra parte, para los tiempos de madurez de $48 \mathrm{~h}$ y $72 \mathrm{~h}$, no se encuentra variabilidad en las edades de 8 y 10 meses de sacrificio, permitiendo predecir un desarrollo fisiológico similar, el cual presenta menor afectación de los cambios bioquímicos postmortem, sobre la CRA. A su vez los resultados de los 10 meses mostraron diferencia significativa en todos los tiempos de madurez con un aumento $23 \%$ en la CRA entre las 24 y las $72 \mathrm{~h}$ de maduración.

\section{CONCLUSIONES}

La edad se muestra como un factor influyente en la capacidad de retención de agua, en donde a mayor edad al momento de sacrificio, se obtiene un menor porcentaje de jugo extraído, por lo tanto mayor
(CRA). El tiempo de maduración estadísticamente mostro que animales jóvenes 4 y 6 meses, presentan disminución en la CRA a mayor tiempo de tenderización. 


\section{REFERENCIAS BIBLIOGRÁFICAS}

Albarracín, W; Sánchez, W. (2013). Caracterización del sacrificio de corderos de pelo a partir de cruces con razas criollas colombianas. Rev. MVZ Córdoba 18(1):3370-3378.

Cañeque, V.; Sañudo, C. Metodología para el estudio de la calidad de la canal y de la carne en rumiantes. Madri: INIA, 2000. 255p.

Chrystall, B.B; Device, C.E. (1985). Electrical stimulation its esrly development in New Zelanda advance in meat research. 1, 73$119 p$.

Diaz Diaz-Chiro M, T. (2001). Características de la canal y de la carne de corderos lechales Manchegos. Correlaciones y ecuaciones de predicción. Tesis doctoral. Universidad Complutense de Madrid. Facultad de Veterinaria. P 43-46.

Hönikel, I. Crowe, P.F.(1998). Reference methods for the assessment of physical characteristics of meat. Meat science, 49(4), 447p.

Huff-Lonergan, E.H; Zhang, W; Lonergan, S.M. (2010). Biochemistry of posmortem muscle lesson on mechanisms of meat tenderization. Meat science. 86, 184-195p.

Huff-Lonergan, E. Lonergan S.M. (2005). Mechanisms of waterholding capacity of meat: the role of postmortem biochemical and structural changes. Meat Science. 71:194-204.

Kim, Y.H; Huff-Lonergan, E; Sebranek, J.G et al; (2010). Highoxygen modified atmosphere packaging system induces lipid and myoglobin oxidation and protein polymerization. Meat Science 85(4) 759-767.

Kuber, P.S; Duckett, S.K; Busboom, J.R; Snowder, G.D; Doobon, M.V; Vierck, J.L et al., (2003). Measuring the effects of phenotype and mechanical restraint on proteolytic degradation and rigor shortening in callipyge lambed muscle during extended ageing. Meat science 63: 325-331p.

Lawrie R.A, Ledward D.A. (2006). Lawrie's meat science. 7th ed. Cambridge, England: Woodhead Publishing. 442 p.
Lawrie, R.A. (1998). Ciencia de la carne, Zaragoza. España. $367 p$.

Martínez-Cerezo, S.; Sañudo, C.; Olleta, J.L; Mendel, I.; Panea, B; Macie, S. et al., (2002). Breed, weight and ageing effects on meat lamb tenderness assessed by consumers. In: 48 th ICoMST. Rome. Vol I: 142-143p.

Melody, J.L.; Lonergan, S.M.; Rowe, L.J. et al., (2004). Early postmortem biochemical factors influence tenderness and water-holding capacity of three porcine muscles. Journal of Animal Science 82(4), 1195-1205.

Offer, G.; Trinick, J. (1983). On the mechanism of water-holding in meat: the swelling and shrinkage of myofibrils. Meat Science 8, 245-281.

Onega, E. Miguel, E. Blazques, B. Ruiz de Huidobro, F. (2001). Evolución de algunos parámetros de calidad de la carne de vacuno en los seis primeros días post mortem. ITE, 22, 568$570 p$.

Ouali, A. (1992). Proteolytic and physicochemical mechanisms involved in meat texture development. Biochimie. 74, 251$265 p$.

Plá, M. (2000). Medida de la capacidad de retención de agua. In: Metodología para el estudio de la calidad de la canal y de la carne en rumiante. Ministerio de ciencia y tecnología. 175-179.

Sañudo, C.; Sierra, I. (1982). Estudio de la calidad de la canal y de la carne en animales cruzados Romanov por rasa Aragonesa. I. Descripción y comparación entre los tipos de ternasco y pascual. VII jornadas científicas de la SEOC. Murcia 121-131.

Sentandreu, M.A.; Coulis, G., Ouali, A. (2002). Role of muscle endopeptidases and the inhibitors in meat tenderness. Trends in food science and technology. 13(12),. 400-421p.

Sierra, S.V. (2010). Evolución post-mortem de parámetros indicativos de calidad en carne de vacuno: efecto de la raza y el gen de la hipertrofia muscular. Universidad de Oviedo. 
Weaver, A.D.; Bower, B.C.; Gerrard, D.E. (2009). Sarcomere length influences $\mu$-calpain-mediated proteolysis of bovine myofibrils. Journal of animal science. 87. 2096-2103p.

Zhang S.X., Farouk M.M., Young O.A., Wieliczko K.J., Podmore C. (2005). Functional stability of frozen normal and high $\mathrm{pH}$ beef. Meat Science 69: 765-772.

Zhang, S.X.; Farouk, M.M.; Young, O.A., et al (2005). Funtional stability of fronzen ormal and high $\mathrm{pH}$ beef. Meat Science 69. $\mathrm{p}$ 765-772.

Weaver, A.D.; Bower, B.C.; Gerrard, D.E. (2009). Sarcomere length influences $\mu$-calpain-mediated proteolysis of bovine myofibrils. Journal of animal science. 87. 2096-2103p.

Wismer-Pedersen, J. (1994). Química de los tejidos animales. En: Ciencia de la carne y los productos cárnicos. 125-149. Ed. J.F. Price, B.S.Schweigert. Acribia. Zaragoza. España. 